\title{
Estimating the Real-Time Respiratory Rate from the ECG with a Bank of Notch Filters
}

\author{
Leila Mirmohamadsadeghi, Jean-Marc Vesin
}

Applied Signal Processing Group, Swiss Federal Institute of Technology, Lausanne, Switzerland

\begin{abstract}
The respiratory rate is an important vital sign that needs to be monitored continuously in clinical and non-clinical health monitoring applications. It is commonly estimated from electrocardiogram (ECG)-derived respiratory waveforms such as the respiratory sinus arrhythmia (RSA) and the ECG R peak amplitudes (RPA). Current methods combine respiratory information from these two waveforms but produce large delays in estimating the respiratory rate. In this work, the powers of the outputs of a bank of order-3 FIR notch filters were used in a recursive scheme to estimate in real-time, and with a small delay, the respiratory rate from the RSA and the RPA waveforms simultaneously. The algorithm was tested on the public Physionet Fantasia data set and compared to the state-of-the-art in terms of estimation accuracy and delay. It was shown that the proposed method provides more accurate estimates with smaller delays than those of the state-of-the-art.
\end{abstract}

\section{Introduction}

The respiratory rate is an important physiological indicator. It is unfortunately not easily recorded in a continuous manner in clinical situations because of the inconvenience of the recording apparatus [1,2]. Furthermore, with the advent of portable health monitoring systems such as smart-shirts, producing a robust and real-time estimate of the respiratory rate is gaining interest for non-clinical applications $[3,4]$. The respiratory rate can be estimated from the electrocardiogram (ECG), as the respiration modulates different aspects of the cardiac activity through neural, physical and reflex processes [5]. ECG-derived respiratory (EDR) waveforms are commonly used to estimate the respiratory rate $[6,7]$. In particular, the respiratory sinus arrhythmia (RSA) waveform, which is the modulation of the cardiac rhythm by the respiration, and the R-peak amplitudes (RPA) waveform, which is the modulation of the ECG amplitude by the respiration, are often used to this end [5]. The combination of several EDR waveforms has been proposed as well in a scheme considering the most dominant spectral peak of all the EDR waveforms as the respiratory rate [7]. However, one major shortcoming of spectral methods is the need to perform computations in a time-window, which introduces long delays in real-time monitoring. Recently, the adaptive multi-signal oscillatorbased band pass filtering (W-OSC) algorithm was proposed as an instantaneous and real-time method of estimating the respiratory rate from two EDR waveforms [6]. This algorithm has a delay of about 10 seconds in tracking the respiratory rate on the Physionet Fantasia data set $[8,9]$. The goal of the present study was to propose a frequency estimation algorithm, capable of processing with low delay and resources multiple EDR waveforms to estimate the respiratory rate. The proposed algorithm uses a bank of order-3 FIR notch filters to probe for the main frequency of the inputs by using the power of the filter outputs in a combined and recursive scheme. Given the inherent low delay of the chosen FIR filters, the developed method is a good candidate in estimating the real-time respiratory rate in health monitoring endeavors. The algorithm was evaluated on the Physionet Fantasia data set and compared to the W-OSC algorithm in terms of estimation error and delay.

\section{Methods}

\subsection{Data}

Data set: The Physionet Fantasia data set was used to evaluate the algorithm. This data set contains 2-hour long simultaneous recordings of the single-lead ECG and the spontaneous respiration (pneumography) acquired from 20 young (21-34 years) and 20 elderly (68-85 years) subjects. The subjects lay supine and watched the "Fantasia" movie while their physiological signals were being recorded. All signals have a sampling rate of $250 \mathrm{~Hz}$.

ECG-derived respiratory waveforms: The ECG R peaks were extracted with a classic extrema-detection method. The $\mathrm{R}$ peak-to-peak time series was created and resampled uniformly at $4 \mathrm{~Hz}$ using cubic spline interpolation. The RSA waveform was created by band-pass filtering the re-sampled $\mathrm{R}$ peak-to-peak series within the respiratory frequencies, i.e., between $0.1-0.5 \mathrm{~Hz}$, using a Butter- 
worth filter. The $\mathrm{R}$ peak amplitudes were also re-sampled similarly to the $\mathrm{R}$ peak-to-peak series and filtered in the same manner to obtain the RPA waveform [6]. The proposed respiratory rate estimation algorithm was applied to the RSA and the RPA, and its estimate was compared to a reference respiratory rate computed from the respiration signal.

Reference respiratory rate: The respiration signal was re-sampled at $4 \mathrm{~Hz}$ similarly to the RSA and the RPA and high pass filtered above $0.1 \mathrm{~Hz}$ using a Butterworth filter. Its instantaneous frequency was computed as the mean output of several classic frequency estimation methods, similarly to [6].

\subsection{Algorithm}

The output of a notch filter is smallest for an oscillation at the notch frequency. The main idea behind the presented algorithm is to use a bank of notch filters to probe the input signal for its main frequency. Small filter output powers indicate the notch frequencies to which the dominant frequency is close. Therefore, they are used in a weighted sum of the notch frequencies to estimate the dominant frequency of the input. As this scheme was designed to be low-delay, it was chosen to use order-3 FIR notch filters characterized by a pair of complex-conjugate zeros with transfer functions $H$ :

$$
H(z)=1-2 z^{-1} \cos \left(2 \pi f_{i}\right)+z^{-2},
$$

where $f_{i}$ is a discrete frequency from a given frequency band $f b=\left[f_{1}, \ldots f_{N}\right]$, with $N$ the number of discrete frequencies. The magnitude response of several such filters is shown in Figure 1. At sample $n$, the output of the $i^{t h}$ filter is:

$$
y_{i}[n]=u[n]-2 u[n-1] \cos \left(2 \pi f_{i}\right)+u[n-2],
$$

where $u[n]$ is the input signal. When the main frequency content of $u$ is close to $f_{i}$, then the output of the notch filter, i.e., $y_{i}$, is small. The output power was computed in a recursive manner, for each filter output, with a forgetting factor $0<\delta<1$ as:

$$
P_{i}[n]=\delta P_{i}[n-1]+(1-\delta) c_{i}^{2}[n],
$$

where $c_{i}$ is a normalized criterion computed from the power of the filter output with respect to the power of the filter input as:

$$
c_{i}[n]=y_{i}[n] / \sqrt{U[n]},
$$

with

$$
U[n]=\delta U[n-1]+(1-\delta) u^{2}[n],
$$

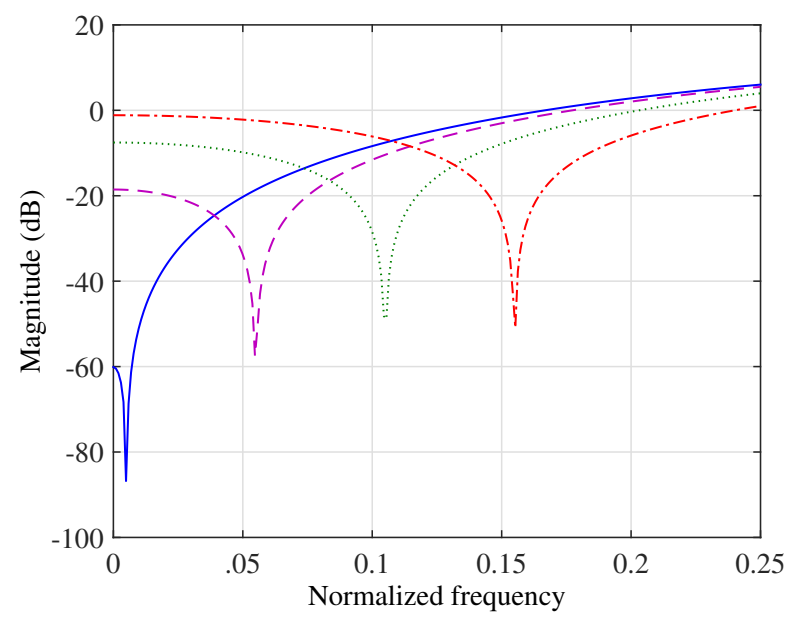

Figure 1. FIR notch filters with transfer functions according to (1).

the recursive estimate of the mean squared-value of the input. The set of $P_{i}$ with $i=1, \ldots N$ were used to compute a set of weights. For each input $u_{j}, j=1, \ldots N$ sig with $N$ sig the number of input signals, (3) yields one set of $P_{i}$. In order to obtain a single weight per frequency $f_{i}$, the $P_{i}$ values were averaged across the inputs $j=1, \ldots N$ sig to create the weights $w_{i}$ :

$$
w_{i}[n]=\frac{1}{N s i g} \sum_{j=1}^{N s i g} P_{i, j}[n] .
$$

Note that the contributions of the different signals do not depend on their respective absolute powers, due to the normalization in (4). It was necessary to scale the weights to give more importance to frequencies yielding small powers and little-to-no importance to those with larger powers. To this end, an exponential weight dissociation scheme was employed, resulting in the new weights $W_{i}$ :

$$
W_{i}[n]=\exp \left(-\gamma[n] w_{i}[n]\right)
$$

where $\gamma$ is a weight dissociation parameter. It was set to the minimum value of $P_{i, j}[n]$ at every sample $\gamma[n]=$ $\min _{i=1, \ldots N}\left(P_{i, j}[n]\right)$.

The final frequency estimate was computed as the weighted sum of the notch frequencies of the filter bank:

$$
f[n]=\frac{\sum_{i=1}^{N} W_{i}[n] f_{i}}{\sum_{i=1}^{N} W_{i}[n]} .
$$

\subsection{Evaluation}

The presented algorithm was applied to the RSA and the RPA as input signals with $f b=[0,0.2], \delta=0.95$ and 
$N=50$, which provides a reasonable resolution in the respiratory frequency band. The sensitivity of the proposed algorithm to its parameters $\delta$ and $N$ was qualitatively investigated on several case records. Systematic evaluation of the algorithm and comparison to the W-OSC results was performed on the entire data set. The parameters of the WOSC algorithm were set as follows: $\beta=0.95, \delta=0.95$ and $\mu=0.95$, which yield a good compromise between the filter bandwidth and the adaptation capability of the algorithm [6]. The initial frequency was set to $f_{0}=0$ as the proposed algorithm does not require an initial frequency.

The estimation error, in terms of the mean absolute error (MAE) in breaths-per-minute (bpm) and the estimation delay (in seconds) of the two algorithms were computed. The estimation delay was evaluated by computing the correlation of the estimate and the reference respiratory rate for various delay values by means of the Pearson correlation coefficient. The delay yielding the largest correlation was selected as the optimal delay for each record.

\section{Results}

Illustrative example: Examples of the RSA and RPA waveforms extracted from the ECG are shown in Figure 2 (a). The respiratory rate estimated by the proposed method, the W-OSC estimate and the reference respiratory rate are presented in Figure 2 (b). It can be seen that the estimate of the proposed method is more accurate and follows the reference rate with less delay. For example, around $t=220 \mathrm{~s}$, there is a marked increase in the reference, which is followed a little later in time by the estimate of the proposed method and even later by the W-OSC estimate. In some portions, the proposed method does not exhibit any delay, such as around $t=280 \mathrm{~s}$. The presence of delay was not systematic and depends on local signal characteristics.

Sensitivity analysis: The sensitivity of the algorithm to $\delta$ and $N$ was qualitatively assessed on a portion of the data set. Figure 3 shows estimates computed with different values of $\delta$ ranging from 0.9 to 0.99 . Figure 4 shows estimates computed with different values of $N$ ranging from 20 to 80 . For a value of $\delta$ closer to 1 , the estimate was less variable but also followed changes in the frequency in more slowly. The value of $N$ did not have a large effect on the estimate. In fact, estimates with $N=50$ and $N=80$ were visually the same.

Overall results: The mean values of estimation errors and delays for both algorithms over the entire data set are reported in Table 1. For both the young and elderly populations, the proposed method yielded lower errors than the W-OSC method. For the proposed method, similarly to the $\mathrm{W}$-OSC, the errors for the young population were smaller than those for the elderly. In terms of estimation delay,
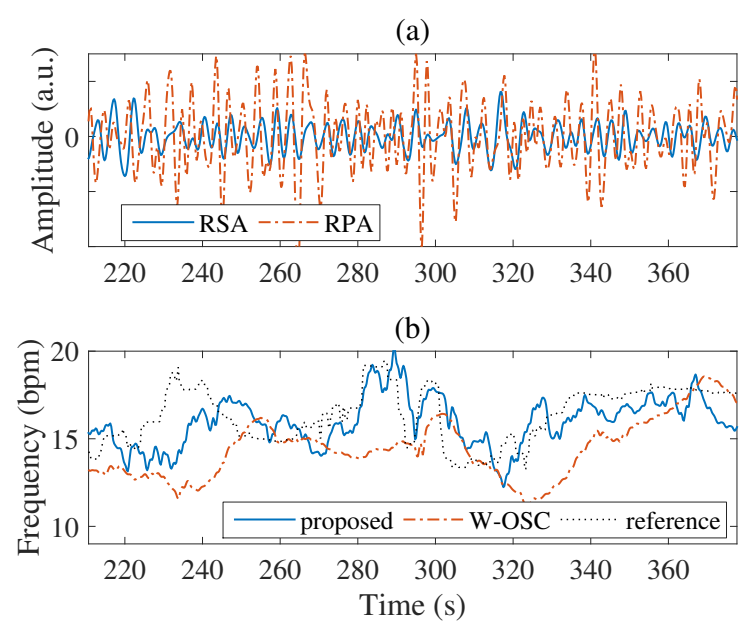

Figure 2. (a) The RSA and RPA, (b) the respiratory rate estimate of the proposed algorithm, that of the W-OSC method and the reference respiratory rate.

the proposed method presented on average half that of the W-OSC. There was no marked difference between the delay in the young and elderly estimates for the proposed method.

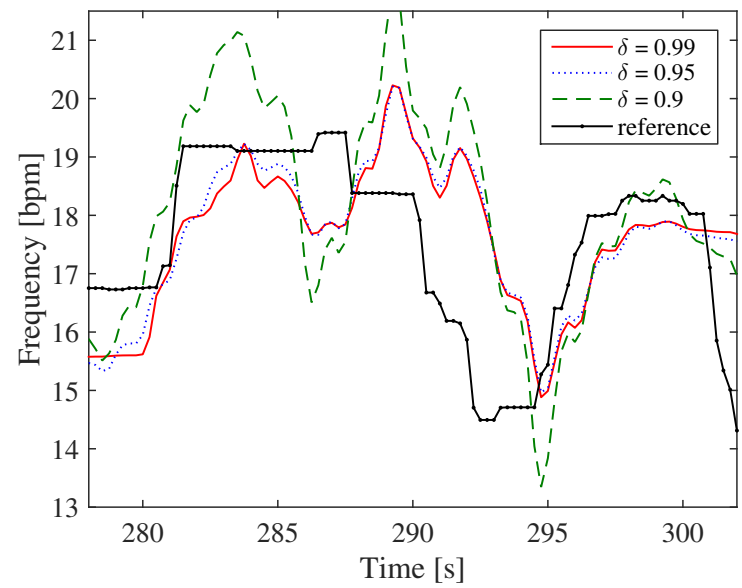

Figure 3. The estimate of the proposed method for several values of the parameter $\delta . N=50, f b=[0,0.2]$.

\section{Discussion and Conclusions}

Although no systematic testing was performed to assess the algorithm sensitivity to its parameters $\delta$ and $N$, qualitative assessment indicated that the parameter $\delta$ had a large influence on the estimate variability but the parameter $N$ did not have a marked effect on the estimate, meaning that the number of discrete frequencies does not need to be very high to achieve acceptable resolution in the estimate. The estimate computed with $N=20$ was slightly more quantized than those computed with $N=50$ and $N=80$ 


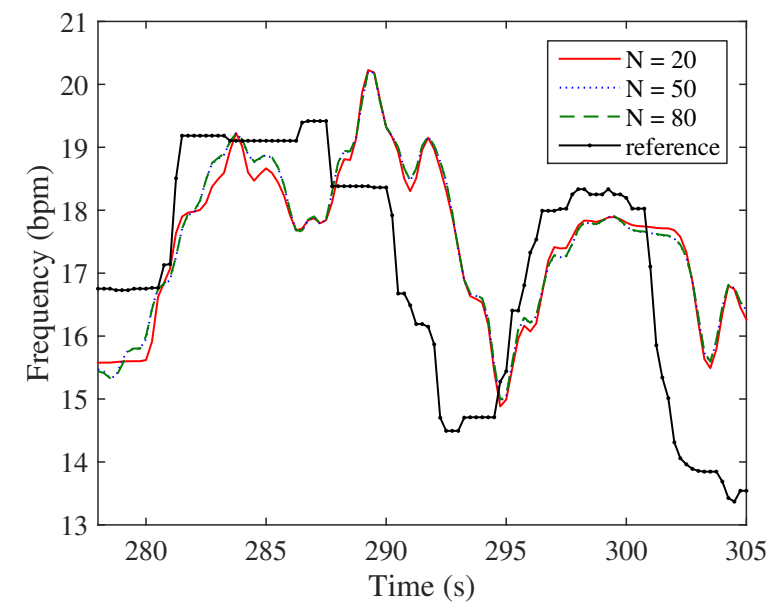

Figure 4. The estimate of the proposed method for several values of the parameter $N . \delta=0.95, f b=[0,0.2]$.

Table 1. MAE (bpm) and estimation delay (s) of the proposed algorithm and the W-OSC algorithm over the Fantasia data set.

\begin{tabular}{lcccc}
\hline \hline & \multicolumn{2}{c}{ Young } & \multicolumn{2}{c}{ Elderly } \\
& MAE & Delay & MAE & Delay \\
\cline { 2 - 5 } W-OSC & 2.65 & 10.75 & 3.17 & 9.5 \\
\hline proposed & 2.63 & 5.25 & 3.11 & 5.25 \\
\hline \hline
\end{tabular}

but between the later two, there was no marked difference. However, larger values of $N$ require more computations. Small values of $\delta$ resulted in large variations in the estimate. Large values prevented the fast adaptation of the estimate to the changes in the input. It was necessary, for each parameter, to select a value providing an acceptable compromise. With the empirically chosen parameters, on the Fantasia data set, the proposed algorithm was better in terms of accuracy than the W-OSC algorithm, although not drastically. In terms of estimation delay, the proposed algorithm provided estimates with half the delay of the WOSC algorithm. This decrease in delay is due to the fact that there are less recursive computations in the proposed algorithm, which uses short FIR notch filters compared to the W-OSC, which uses an IIR band-pass filter. In the proposed algorithm, only the weights of the discrete frequency values serving as filter centers are computed in a recursive manner. In the W-OSC method, however, both the filter central frequency and the weights of the various inputs are computed in a recursive manner. It must be noted that to reduce the total delay of the real-time implementation, linear interpolation must be used instead of cubic spline interpolation in the re-sampling of the EDRs.

In this paper, evaluation was directly performed on a set of real data. A detailed study of the proposed algorithm in terms of the estimation bias and variance and their sensitivity to the algorithm parameters must be carried out on simulated data.

This algorithm is a promising tool to provide easily computed, accurate real-time respiratory rate estimates from a single-lead ECG in a clinical setting and also on portable health monitoring platforms.

\section{Acknowledgments}

This work was funded thanks to the Swiss NanoTera initiative, RTD project ObeSense.

\section{References}

[1] Cretikos MA, Bellomo R, Hillman K, Chen J, Finfer S, Flabouris A. Respiratory rate: the neglected vital sign. Med J Aust 2008;188(11):657.

[2] AL-Khalidi FQ, Saatchi R, Burke D, Elphick H, Tan S. Respiration rate monitoring methods: A review. Pediatr Pulmonol 2011;46(6):523-529.

[3] Schleusing O, Renevey P, Bertschi M, Dasen S, Paradiso R. Detection of mood changes in bipolar patients though monitoring of physiological and behavioral signals. In European Conference of the International Federation for Medical and Biological Engineering. 2012; 1106-1109.

[4] Mirmohamadsadeghi L, Fallet S, Buttu A, Saugy J, Rupp T, Heinzer R, Vesin JM, Millet GP. Sleep apnea detection using features from the respiration and the ECG recorded with smart-shirts. In Biomedical Circuits and Systems Conference (BioCAS). IEEE, 2014; 61-64.

[5] Bailon R, Sornmo L, Laguna P. Ch 8: ECG- Derived Respiratory Frequency Estimation. Advanced Methods and Tools for ECG Data Analysis, 1 edition. Norwood, MA, USA: Artech House, 2006; .

[6] Mirmohamadsadeghi L, Vesin JM. Respiratory rate estimation from the ECG using an instantaneous frequency tracking algorithm. Biomed Signal Process Control 2014;14(0):6672.

[7] Orphanidou C, Fleming S, Shah SA, Tarassenko L. Data fusion for estimating respiratory rate from a single-lead ECG. Biomed Signal Process Control 2013;8(1):98-105.

[8] Goldberger AL, Amaral LA, Glass L, Hausdorff JM, Ivanov PC, Mark RG, Mietus JE, Moody GB, Peng CK, Stanley HE. PhysioBank, PhysioToolkit, and PhysioNet: components of a new research resource for complex physiologic signals. Circulation 2000;101(23):215-220.

[9] Iyengar N, Peng C, Morin R, Goldberger A, Lipsitz LA. Age-related alterations in the fractal scaling of cardiac interbeat interval dynamics. Am J Physiol 1996;271(4):10781084.

Address for correspondence:

Leila Mirmohamadsadeghi

EPFL SCI STI JMV ELD 234 Station 11

1015 Lausanne, Switzerland 